\title{
Auditory Stimuli as Environmental Enrichment Tool for Family Dogs
}

\author{
Megumi Fukuzawa ${ }^{1} \&$ Shihori Kajino ${ }^{1}$ \\ ${ }^{1}$ Nihon University, College of Bioresource Sciences, Kanagawa, Japan \\ Correspondence: Megumi Fukuzawa, Nihon University, College of Bioresource Sciences, 1866 Kameino, \\ Fujisawa, Kanagawa, 252-0880, Japan. Tel: 81(0)466-34-3655. E-mail: fukuzawa.megumi@nihon-u.ac.jp
}

Received: April 7, 2018

Accepted: April 16, 2018

Online Published: April 27, 2018

doi:10.5539/ijb.v10n3p19

URL: https://doi.org/10.5539/ijb.v10n3p19

\begin{abstract}
Ten healthy pet dogs with an average maximum resting heart rate of $92 \mathrm{bpm}$ that had never been used in studies of auditory stimulation were exposed randomly to one of three sound conditions on each of three occasions within a 5-day period. Posture and behaviour were recorded continuously by video for a total of $20 \mathrm{~min}$ over three phases: 5 min before sound exposure, 10 min during sound exposure, and $5 \mathrm{~min}$ after sound exposure. Each dog wore a Polar HR monitor throughout testing, and heart rate was recorded by using $\mathrm{R}-\mathrm{R}$ interval data. Maximum heart rate was significantly greater during heartbeat sound exposure than afterwards, and average heart rate with both heartbeat sound and classical sound showed decrease tendencies. The time spent in dynamic (e.g. movement-related) postures was significantly higher before treatment than during or after under all three conditions. These data suggest that auditory stimuli in dogs may affect physiological responses without necessarily affecting behaviour, and specifically that heart rate may be elevated by auditory exposure to a faster heartbeat.
\end{abstract}

Keywords: auditory stimulus, behaviour, cognition, dogs, heart rate

\section{Introduction}

Dogs under stress show high levels of activity (e.g., Beerda et al., 2000), including vocalizing and locomotive behaviors. Auditory stimulation can be a useful environmental enrichment for reducing these activity levels in kennelled dogs (e.g. Wells et al., 2002; Kogan et al., 2012), although its value depends on the type of sound. Wells et al. (2002) found that dogs housed in a rescue shelter spent significantly more time being quiet when they were exposed to classical music than when they were exposed to human conversation, heavy metal music, pop music, or no music. Kogan et al. (2012) also reported that classical music causes kennelled dogs to spend more time sleeping and less time vocalizing than when they are exposed to other types of music (i.e. heavy metal or modified classical music designed specifically for dog relaxation), although heavy metal music increased the dogs' body-shaking movements. Bowman et al. (2015) found that dogs became habituated to the calming effects of classical music as early as the second day of exposure; moreover, auditory stimulation induced changes in heart rate variability and behaviour indicative of reduced stress levels. It thus seems that classical music calms kennelled dogs more effectively than do other music types.

Brayley and Montrose (2016) found that exposure to audiobooks also enhanced the welfare of kennelled dogs by increasing their resting behaviour and reducing vocalizing and sitting or standing behaviours. Although sound has been shown to be a useful enrichment tool for dogs, to our knowledge its effects on small groups of dogs have not yet been investigated. Products that have a realistic-feeling heartbeat (e.g. Snuggle Puppy, Smart Pet Love, MI, US) seem to be able to eliminate some negative emotional states such as fear and anxiety, but there is no scientific support for such products. Kawakami et al. (1996) assessed the effects of heartbeat, white noise, and no-sound conditions on newborn infants' behavioural and physiological responses to stress. Both the heartbeat and white noise had a calming effect on these babies. However, the potential of heartbeat sound as an auditory stimulus has not yet been investigated in dogs. If a dog lives at home, it seems that a dog may experience a situation where is sleeping together or being hugged by the owner, owner's family member or a familiar person. Even if the dog had been listening to a dam' or a litter's heartbeat sound at a young age, the chances of listening to human's heartbeat sounds will increase as time spent with human. Therefore, the effects of human heartbeat sounds may expect to family dogs who living with owner's family member as same as newborn babies. Here, we therefore aimed to investigate the influence of the sound of a heartbeat or classical music on dogs. 


\section{Method}

\subsection{Animals}

Ten healthy family pet dogs -3 German shepherds, 1 Border collie, 1 Golden retriever, 1 Shiba-inu, and 4 crossbreds ( 7 females and 3 males) - participated in the study. The dogs varied in age (from 8 to 103 months) and in weight (from 7.1 to $34.2 \mathrm{~kg}$ ). None of dogs had a history of participation in studies of auditory stimulation, and none showed fright or flight behaviour towards sound. All owners or persons who regularly handled the dogs were informed and agreed to attend the study. On the day of the experiment, the dogs were kept individually in their own familiar kennels at the university during the day and were all handled equally. All dogs were allowed contact with the outside environment and with humans at any time while staying at the university to participate in the tests. They returned to their own homes after finishing the experimental work.

\subsection{Auditory Stimulation}

We used two experimental auditory stimulation conditions and one control, namely heartbeat (125 bpm): dogs were exposed to a heartbeat soundtrack chosen from the 'Discovery Sound CD' (Discovery Firm Inc., Hokkaido, Japan; sound volume $35.1 \pm 0.8 \mathrm{db}$, as measured by using an SM-325 sound level meter; As ONE, Osaka, Japan). This heartbeat was that of a human; classical music: dogs were exposed to the Pachelbel's Canon track from the 'Canon Piano Solo CD' (Meister Music Co., Ltd., Kanagawa, Japan; $39.0 \pm 1.3 \mathrm{db}$ ); and control: dogs were exposed to no added auditory stimulation, but the loudspeaker used in the other two conditions (SRS-A3, Sony, Tokyo, Japan) was presented to each dog. The volume was set so that the experimenter confirmed at the centre of the unit $(100 \mathrm{~cm}$ from the speaker) and the volume that the experimenter feels comfortable (e.g. no sound cracking, difficult to hear). Dogs could sometimes hear noise arising naturally from their environment during the presentation of all stimuli.

\subsection{Experimental Room}

The experiment was conducted in a room at the university to which the dogs were habituated over at least a 6 month period (Figure 1). The room was divided into two equal-sized observational units (each $105 \times 214 \mathrm{~cm}$ ); therefore, two dogs were able to be observed at the same time. Three video cameras (DCR-SR87, Sony, Tokyo, Japan) were used to record the dogs' behaviour, and a loudspeaker was located in front of each dog's area. One experimenter and two of the three video cameras were located behind an opaque panel. The third camera was located opposite the other two. This camera was used as an aid when the dog was not visible from the two front cameras. The room temperature was controlled at around $21.0^{\circ} \mathrm{C}$ (mean temperature, $20.6 \pm 0.7^{\circ} \mathrm{C}$ ).

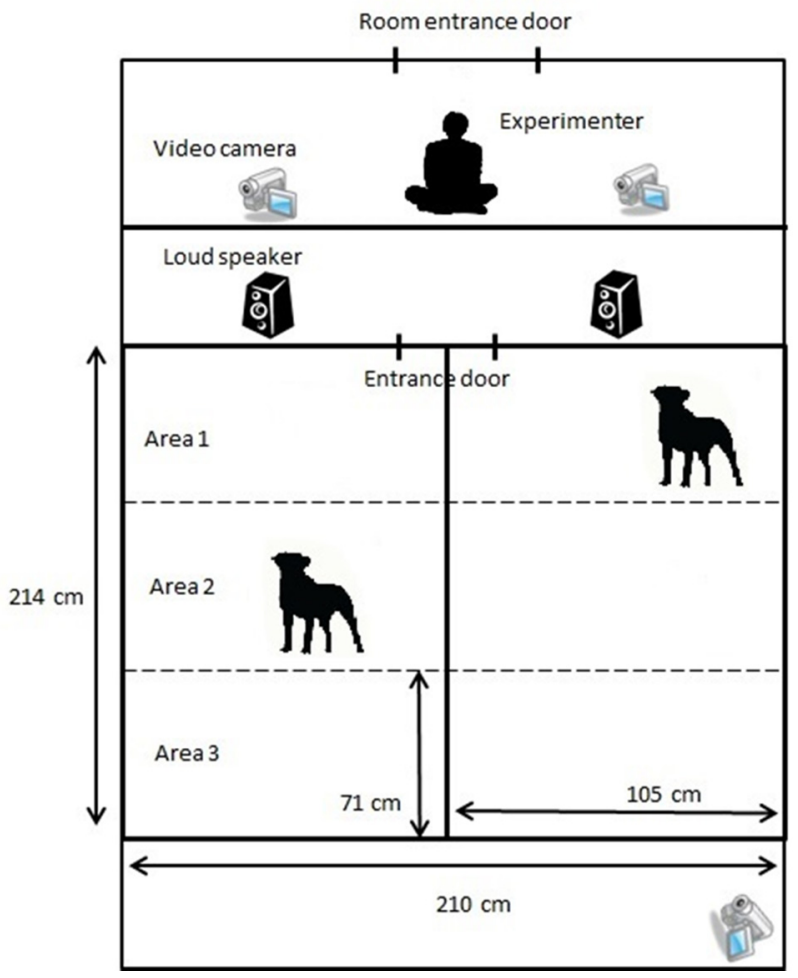

Figure1. Layout of experimental room 


\subsection{Experimental Schedule and Data Collection}

After the dog had been given time to defecate and urinate as needed, it was equipped with a heart rate monitor (RS800CX; Polar Electro Oy, Kampele, Finland) and then brought into the experimental room. The experimenter (a woman in her 20s), who was familiar with all of the dogs, let the dog off its leash, and the dog was then able to spend time freely in its own unit. The experimenter sat on the floor in a fixed position; she was hidden from the dog's sight by a barrier (see Figure 1). After the experimenter had confirmed that the dog's heart rate was within the normal range, the test was started. All tests were conducted between 9:30AM and 4:00PM.

The three conditions (heartbeat, classical music, and control) were presented in random order to each dog on three test days, at one condition per test day, within a 5-day period; the set of three tests was then repeated 1 week, and again 2 weeks, after the end of the previous test period. In other words, each condition was presented only three times during the whole experimental period. Posture (five categories) and behaviour (10 categories) (Table 1) were recorded continuously on video for a total of 20 min over three phases: 5 min before sound exposure, $10 \mathrm{~min}$ during sound exposure, and 5 min after the end of sound exposure. Heart rate was recorded by using R-R interval data to determine the mean, maximum and minimum heart rates during each phase. The time the dog spent positioned towards the loud speaker in the observational unit was also recorded.

Table 1. Dog behaviours recorded

\begin{tabular}{ll}
\hline Observational category & Definition \\
\hline$<$ Posture $>$ & Dog sits on floor \\
Sitting & Dog stands on all four legs \\
Standing & Dog lies down on sternum or curls up into a ball \\
Sternal lying or curling up & Side of dog's body contacts floor \\
Lateral recumbency & Put paws on the equipment, locomotion etc. \\
Dynamic postures & \\
$<$ Behaviour $>$ & Dog barks or growls \\
Barking & Dog whines \\
Whining & Dog pants with open mouth \\
Panting & Dog stretches its forelegs or hind legs \\
Stretching & Dog opens mouth widely and exhales \\
Yawning & Dog pricks up its ears \\
Listening & Dog sniffs the floor \\
Sniffing the floor & Dog eats floor covering or wall partition \\
Pica & Dog tries to escape \\
Escaping & (e.g. scratching or chewing the equipment, or jumping) \\
& Dog digs on the floor \\
\hline
\end{tabular}

\subsection{Statistical Analysis}

An analysis of variance to assess the effect of the experimental conditions on the percentage of time spent in each posture or behaviour or with each heart rate was used. Post-hoc Tukey tests were used for pairwise comparison of the means of the times when significant effects were found. The percentages of time spent in five out of 10 categories (yawning, whining, stretching, pica and escaping) under each condition were low; therefore, these behaviours were excluded from the statistical analysis. ANOVA was also performed on the effects of the three observational phases on the minimum, average, and maximum heart rates during each set of stimulus conditions.

\section{Results}

\subsection{Heart Rate}

The only significant change was in maximum heart rate during heartbeat sound presentation: maximum heart rate was significantly lower after the end of exposure than during exposure (Tukey, $P<0.05$ ). There were decrease tendencies in average heart rate with both heartbeat sound exposure $(P=0.092)$ and classical music exposure $(P=$ 0.068), but non-significant with control $(P=0.105)$ (Figure 2). 


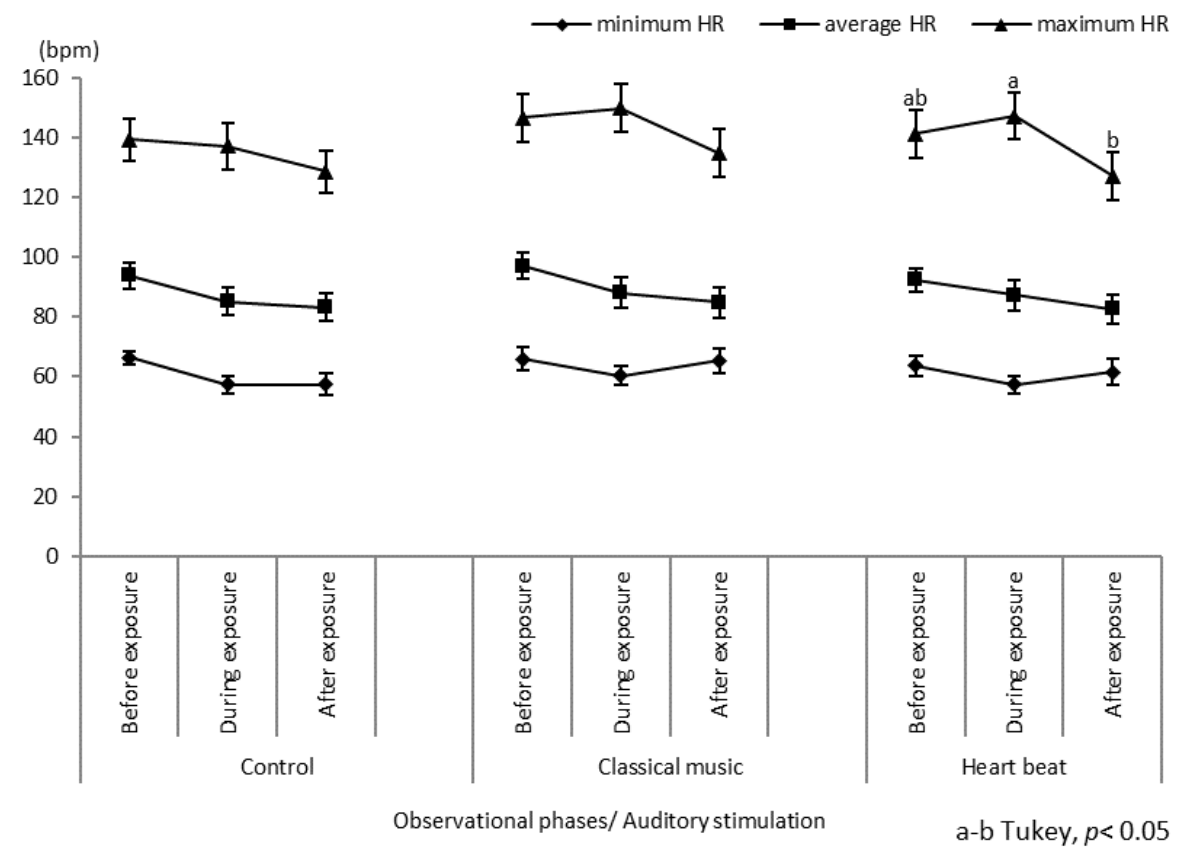

Figure 2. Minimum, average and maximum HR during each period, each stimulus condition

\subsection{Posture}

There were no effects of treatment on percentage of time in each posture but in all cases dogs spent more time in sternal lying than all other positions (Tukey, $P<0.05$ ). Under all three conditions, the time spent in dynamic postures was significantly less in the during- and after- sound-exposure phases than in the before-exposure phase (Tukey, $P<0.05$ ) (Figure 3).

Repeated exposure to heartbeat sound had a significant effect on the amount of time spent in lateral recumbency. The time spent in lateral recumbency $(21.4 \%)$ was significantly greater during the third exposure to the heartbeat track than during both the first $(0.4 \%)$ and second $(1.1 \%)$ exposures (ANOVA, $P<0.05$ ).

\subsection{Behaviours}

The percentage of time spent sniffing the floor was significantly greater before exposure than after exposure under all three conditions, whereas under classical music or heartbeat conditions the percentage of time spent listening was significantly greater during stimulus presentation than before (Tukey, $P<0.05$ ). Neither barking nor panting time was influenced by any of the conditions. Repetition of exposure had no effect on the percentage of time spent in each behaviour (Figure 4). 

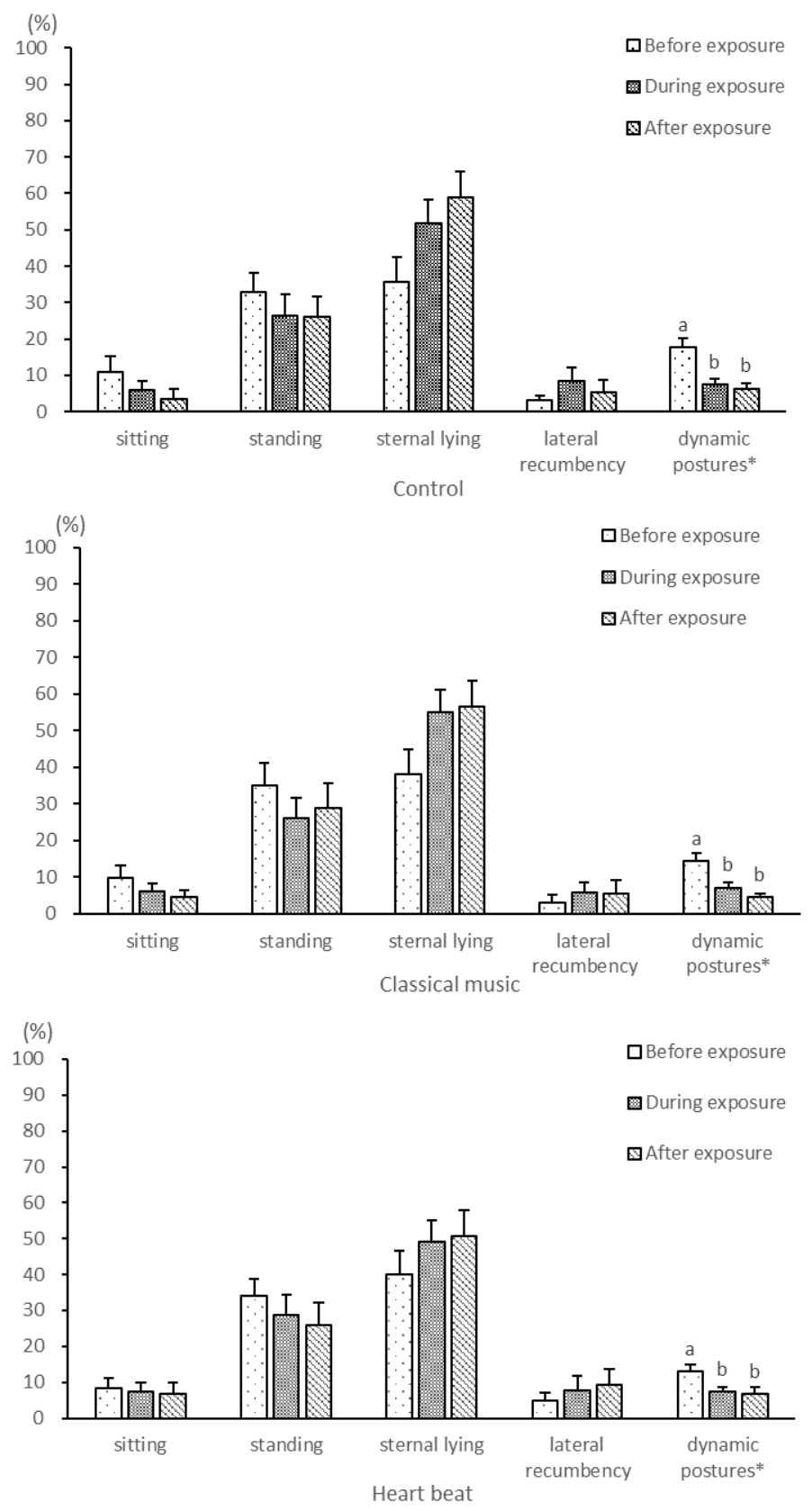

Figure 3. Percentages of time spent in each posture under each condition (top, control; middle, heartbeat sound; bottom, classical music). Different letters a and $\mathrm{b}$ denote significant differences within each posture (Tukey, $P<$ $0.05)$

\subsection{Staying Position}

The percentages of time spent in the three observational areas (see Figure 1) differed significantly. Dogs spent longer in area 1 (closest to the speaker) than both in area 2 and 3 under both classical music and control conditions (Tukey, $P<0.05$ ). Although dogs also spent longest in area 1 both before and during heartbeat exposure (Tukey, $P$ $<0.05$ ), there were no significant differences in time spent in the three areas after exposure to heartbeat sound (ANOVA, $P=0.06$ ). 

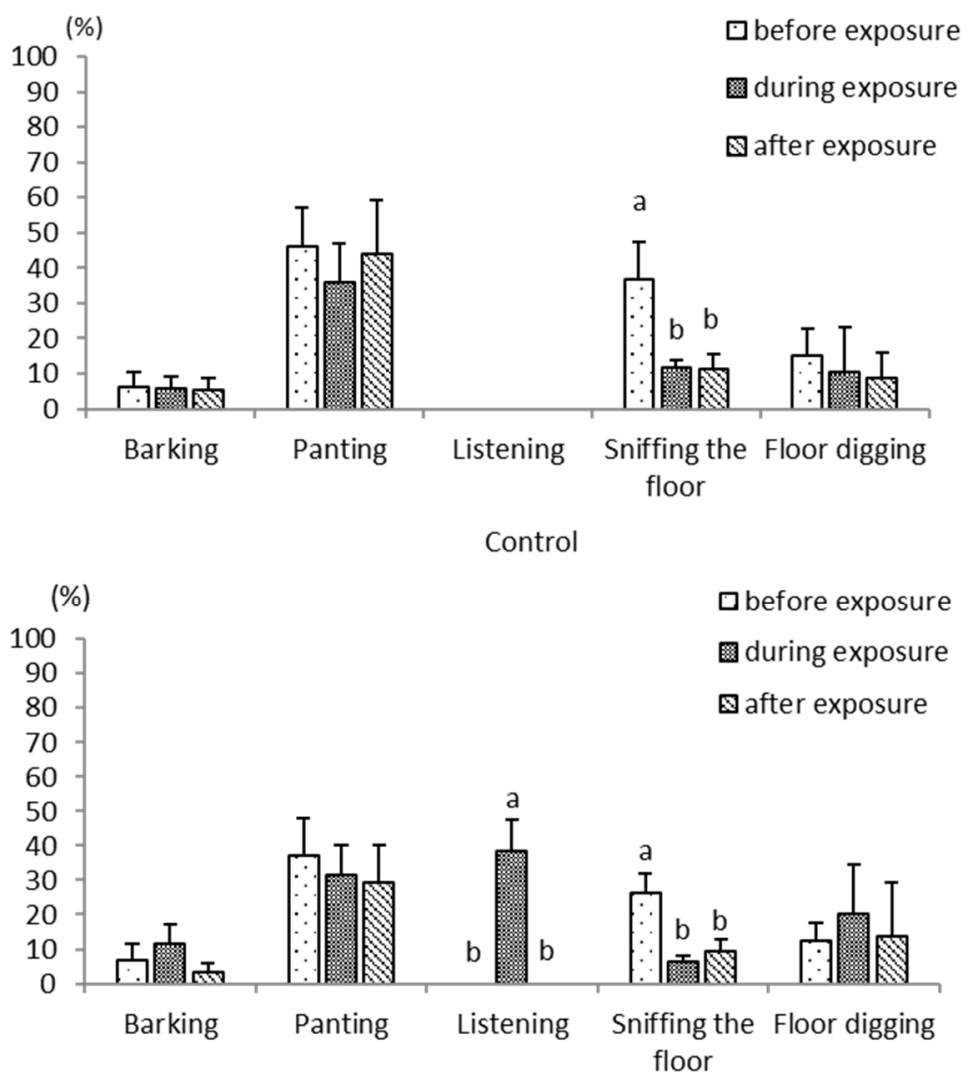

Heart beat

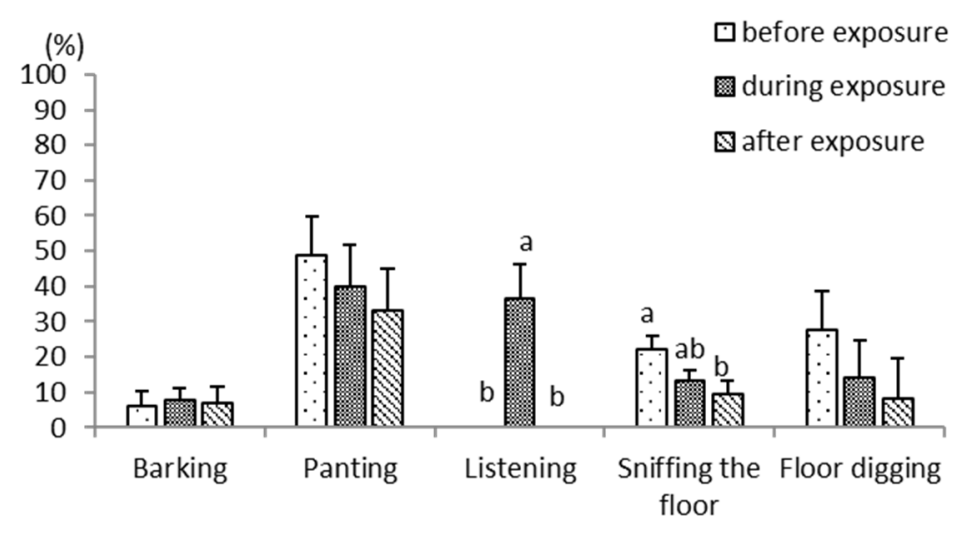

Classical music

Figure 4. Percentages of time spent in each behaviour under each condition (top, control; middle, heartbeat sound; bottom, classical music). Different letters a and $\mathrm{b}$ denote significant differences within each behaviour

(Tukey, $P<0.05$ )

\section{Discussion}

These recorded human beats were more rapid than those of dogs. The human heartbeat fluctuates with a 1/f-like scaling of the frequency spectrum (e.g. Kobayashi and Musha, 1982). The spectrum of $1 / \mathrm{f}$ fluctuation is neither regular nor random; it seems to exist in natural phenomena such as the twittering of birds or the murmuring of people in a comfortable situation. To our knowledge, there has been no scientific report of how $1 / \mathrm{f}$ fluctuation affects the human body, except in the case of somesthesia. The $1 / \mathrm{f}$ noise is also described as pink noise. Pink noise 
differs from white noise: white noise comprises random and equal amounts of frequencies from the entire sound spectrum, whereas pink noise also includes noise from the entire spectrum but emphasizes specific, lower frequencies that create a deeper, gentler sound. Although the effects of heartbeat sound (or pink noise) have not been described in many papers, products that arouse natural instincts, such as Snuggle Puppy, with its realistic pulsing heartbeat, are often described as having a calming influence on animals in marketing materials. In this experiment, a significant difference in maximum heart rate was observed after the heartbeat sound exposure. Human heartbeat sounds may have a calming effect on dogs after playback than during playback. Or perhaps if we play for more than 10 minutes continuously, a calming effect may be recognised during playback. The sound exposure time in the previous studies (e.g. Kogan et al., 2012; Bowman et al., 2015) was longer than that of our experiment, these were done in a dog's unfamiliar environment (e.g. a shelter). In other words, if the dog is well habituated the environment where listen to the sound, it is considered that the effect will start to appear with exposure of about 10 minutes, and it was indicated that both playback time and the environment that let the dog hear the sound can be an important factor.

The dogs' levels of movement were relatively constrained during and after exposure to all three conditions, because the time spent in dynamic postures was less than that spent in the other postures. The results suggested that the dogs' activity levels, as indicated by their behaviour, increased when they shifted sites from their own day kennels to the experimental room, even if they had been habituated to the environment of the experimental room. However, under all conditions the percentage of time spent in sternal lying during each phase was significantly greater than that spent in other postures. Therefore, before the start of an experimental session, it may be appropriate to pre-habituate the dog to the new site for at least $5 \mathrm{~min}$ under control conditions, because their activity levels are more stable if they have been habituated.

Only the percentage of time spent in lateral recumbency during heartbeat exposure increased with repetition. The utility of classical music has also been reported in dogs (e.g., Wells et al., 2002; Kogan et al., 2012; Bowman et al., 2015), but the exposure periods used were longer (from 45 to $390 \mathrm{~min}$ ) than our tests (10 min). It is obvious that classical music is a particularly efficient tool for enriching the environments of animals, although there are differences in efficacy among music types and exposure times. An abundance of time spent in resting postures such as sternal lying, sitting and lateral recumbency was observed by us and these other researchers under all conditions; therefore, the effect may not be restricted to classical music. However, perhaps, as we found here, the effect of repetition is associated only with heartbeat sounds. Salk (1973) points out that a mother's heartbeat sound brings psychological pleasure to an infant, likely because of the baby's memory of conditions inside the womb. Dogs may respond similarly to infants to the human adult heartbeat sound. Even with a short exposure time, our results suggested that the effect was apparent (and cumulative) more than 3 weeks after the first experience, although further research in this regard is needed.

Sound volume has not been addressed in many reports. Kawakami and colleagues (1996) presented either heartbeat sound or white noise at $85 \mathrm{db}$ to newborn infants; they found that the volume was similar inside and outside the womb. Although the distance from the sound source affects the perceived volume, a volume of between 80 and $90 \mathrm{db}$ seems unbearable in situations such as inside a train carriage, or a short distance from a full-volume television or a barking dog. Both heartbeat and white noise conditions were more effective in reducing stress in 5-day-old infants than were no-sound conditions, despite the use of these sound volumes. However, in our study, both the heartbeat sounds and the classical music were presented to the dogs at about $35 \mathrm{~dB}$, as measured within 1 $\mathrm{m}$ from the speaker. This volume level is similar to that of whispering. This may have been the reason why the dogs stayed mainly in area 1 during exposure. If the sound level were strengthened and the dogs' exposure to it increased, we may have obtained different results.

\section{Conclusions}

Auditory stimulation with either heartbeat sounds or classical music may yield a greater calming response than control conditions in dogs. The dogs' heart rates may be elevated during exposure to the sound of a faster heart than their own.

\section{References}

Beerda, B., Schilder, M. B., Van Hooff, J. A., De Vries, H. W., \& Mol, J. A. (2000). Behavioural and hormonal indicators of enduring environmental stress in dogs. Animal Welfare, 9, 49-62.

Bowman, A., Scottish, S. P. C. A., Dowell, F. J., \& Evans, N. P. (2015). 'Four Seasons' in an animal rescue centre; classical music reduces environmental stress in kennelled dogs. Physiology \& Behavior, 143, 70-82. http://doi.org/10.1016/j.physbeh.2015.02.035 
Brayley, C., \& Montrose, V. T. (2016). The effects of audiobooks on the behaviour of dogs at a rehoming kennels. Applied Animal Behaviour Science, 174, 111-115. https://doi.org/10.1016/j.applanim.2015.11.008

Kawakami, K., Takai-Kawakami, K., Kurihara, H., Shimizu, Y., \& Yanaihara, T. (1996). The effect of sounds on newborn infants under stress. Infant Behavior and Development, 19(3), 375-379. https://doi.org/10.1016/S0 163-6383(97)90041-2

Kobayashi M., \& Musha T. 1982. 1/f fluctuation of heartbeat period. IEEE transactions on biomedical engineering, 29, 456-457. https://doi.org/10.1109/TBME.1982.324972

Kogan, L. R., Schoenfeld-Tacher, R., \& Simon, A. A. (2012). Behavioural effects of auditory stimulation on kennelled dogs. Journal of Veterinary Behevior, 7, 268-275. https://doi.org/10.1016/j.jveb.2011.11.002

Salk, L. (1973). The role of the heartbeat in the relations between mother and infant. Scientific American, 228, 24-29.

Wells, D. L., Graham, L., \& Hepper, P. G. (2002). The influence of auditory stimulation on the behaviour of dogs housed in a rescue shelter. Animal Welfare, 11, 385-393.

\section{Copyrights}

Copyright for this article is retained by the author(s), with first publication rights granted to the journal.

This is an open-access article distributed under the terms and conditions of the Creative Commons Attribution license (http://creativecommons.org/licenses/by/4.0/). 